

\section{Giriş}

Bireyler, yatırımcılar ve şirketler yaşamları süresince karar verirler. Bu kararların sağladığı fayda katlanılan maliyetin üzerinde gerçekleştiğinde bir değer yaratılmış olur. Verilen her karar karşılaşılan bir problemin bir çözümünü gösterir. Eğer, gerçek dünya tam bilgi varsayımı altında çalışıyor olsaydı alınan her karar doğru olacak ve günlük hayat optimal seçimlerden oluşacaktır. Buna karşılık, günlük hayat eksik bilgi gerçeği altında hareket etmektedir. Piyasaya gelen her yeni bilgi varlıkların fiyatlamasını açıklamaktadır. Bu bilgiler piyasa tarafından bazen olumlu algılanacağı gibi bazen de olumsuz algılanmaktadır. Piyasa oyuncuları bu risk unsurlarına bağlı olarak pozisyon alırlar.

Bireyler ve şirketler karar verme aşamasında çok sayıda alternatif ve kriteri analiz etmek zorunda kalırlar. Çok sayıda alternatif ve kriterlerden oluşan bir karar probleminde Çok Kriterli Karar Verme (MCDM) yöntemleri uygulanır. Bu yöntemler her zaman optimal bir çözüm önermez. Ancak, olası karar hatalarını en aza indirirler.

COmplex PRoportional ASsessment (COPRAS) çok kriterli karar verme yöntemlerinden biridir. (Podvezko, 2011; Das, 2012; Amoozad Mahdiraji, vd., 2018; Roozbahani, vd., 2020) göre, ideal ve en kötü-ideal çözümleri göz önünde bulundurarak en iyi karar alternatiflerini seçer. Alternatiflerin önemi ve fayda derecesi açısından aşamalı bir sıralama ve değerlendirme prosedürü uygular. Kriter değerlerini hem maksimize etme hem de minimize etmek için çok kriterli değerlendirme yaklaşımı kullanılır. Yöntem, kriterleri maksimize etmenin ve en aza indirmenin değerlendirme sonucuna etkisini inceler. En iyi alternatifin seçimi, hem ideal hem de ideal olmayan çözümler dikkate alınarak yapılır. Mevcut senaryolar arasından en iyi senaryoyu seçer.

Yönteminin güvenilirliği ve doğruluğu birçok bilim insanı tarafından kabul edilmektedir ve günümüzde farklı mühendislik ve yönetim alanlarında çok özellikli problemleri çözmek için kullanılmaktadır. Yöntemin hesaplama aşamaları ve süresi kısadır. COPRAS yöntemi alan yazınına (Zavadskas, vd., 1994) tarafından kazandırılmıştır.

\section{Literatür Taraması}

COPRAS yöntemiyle yapılan çalışmalar Tablo 1'de özetlenmiştir.

Tablo 1: COPRAS Yöntemiyle Yapılmış Çalışmalar

\begin{tabular}{|l|l|l|l|l|}
\hline No & Yazarlar & Çalışmanın Türü & Çalışmanın Amacı & Sonuç \\
\hline $\mathbf{1 .}$ & $\begin{array}{l}\text { (Kaklauskas, vd., } \\
\text { 2006) }\end{array}$ & Vaka çalışmasıdır. & $\begin{array}{l}\text { Bina bakım oranımı konusunda } \\
\text { müteahhit seçimidir. }\end{array}$ & $\begin{array}{l}\text { COPRAS yöntemi bu seçimi } \\
\text { yapabilir. }\end{array}$ \\
\hline $\mathbf{2 .}$ & $\begin{array}{l}\text { (Zavadskas, vd., } \\
\text { 2007) }\end{array}$ & Vaka çalışmasıdır. & $\begin{array}{l}\text { Yol yapımında alternatiflerin } \\
\text { değerlendirilmesidir. }\end{array}$ & $\begin{array}{l}\text { Araştırma, COPRAS } \\
\text { yönteminin kullanım için } \\
\text { uygun olduğu sonucuna } \\
\text { varmıştır. }\end{array}$ \\
\hline $\mathbf{3 .}$ & $\begin{array}{l}\text { (Zagorskas, vd., } \\
\text { 2007) }\end{array}$ & Vaka çalışmasıdır. & $\begin{array}{l}\text { Şehirleşme ilkelerinin } \\
\text { uygulanması yoluyla } \\
\text { sürdürülebilir kalkınmanın } \\
\text { sağlanmasıdır. }\end{array}$ & $\begin{array}{l}\text { COPRAS yöntemi şehir } \\
\text { planlama yetkililerine ve şehir } \\
\text { ylanlama uzmanlarına, } \\
\text { yardımcı olacaktır. }\end{array}$ \\
\hline $\mathbf{4 .}$ & $\begin{array}{l}\text { (Zavadskas, vd., } \\
\text { 2008) }\end{array}$ & Vaka çalışmasıdır. & $\begin{array}{l}\text { İnşaat proje yöneticisi } \\
\text { seçimidir. }\end{array}$ & $\begin{array}{l}\text { COPRAS-G yöntemi bu } \\
\text { seçimi yapabilir }\end{array}$ \\
\hline $\mathbf{5 .}$ & (Podvezko, 2011) & Vaka çalışmasıdır. & $\begin{array}{l}\text { Çok kriterli karar verme } \\
\text { SAW ve COPRAS aynı }\end{array}$ \\
\hline
\end{tabular}




\begin{tabular}{|c|c|c|c|c|}
\hline & & & $\begin{array}{l}\text { yöntemlerinden ikisinin } \\
\text { karşılaştırılmasıdır. }\end{array}$ & $\begin{array}{l}\text { hiyerarşik seviyedeki } \\
\text { büyüklüklerin } \\
\text { karşılaştırılmasını yapabilirler. }\end{array}$ \\
\hline 6. & $\begin{array}{l}\text { (Kildienè, vd., } \\
\text { 2011) }\end{array}$ & Vaka çalışmasıdır. & $\begin{array}{l}\text { İnşaat sektörü ve farklı } \\
\text { ülkelerdeki ülke ekonomisine } \\
\text { etkisidir. }\end{array}$ & $\begin{array}{l}\text { COPRAS yöntemi inşaat } \\
\text { sektörünü tanımlayan } \\
\text { kriterleri değerlendirebilir. }\end{array}$ \\
\hline 7. & $\begin{array}{l}\text { (Yazdani, vd., } \\
\text { 2011) }\end{array}$ & Vaka çalışmasıdır. & $\begin{array}{l}\text { Hidrokarbon tank kamyon } \\
\text { taşıma sisteminin seçimidir. }\end{array}$ & $\begin{array}{l}\text { Fuzzy COPRAS yöntemi bu } \\
\text { seçimi yapabilir. }\end{array}$ \\
\hline 8. & $\begin{array}{l}\text { (Chatterjee, vd., } \\
\text { 2011) }\end{array}$ & Vaka çalışmasıdır. & $\begin{array}{l}\text { En uygun malzemenin } \\
\text { seçimidir. }\end{array}$ & $\begin{array}{l}\text { COPRAS ve EVAMIX } \\
\text { yöntemleri bu seçimi } \\
\text { yapabilir. }\end{array}$ \\
\hline 9. & $\begin{array}{l}\text { (Popovic, vd., } \\
\text { 2012) }\end{array}$ & Vaka çalışmasıdır. & Yatırım projesinin seçilmesidir. & $\begin{array}{l}\text { En iyi proje COPRAS ve } \\
\text { COPRAS-G yöntemleri } \\
\text { kullanılarak seçilebilir. }\end{array}$ \\
\hline 10. & (Zolfani, vd., 2012) & Vaka çalışmasıdır. & Personel seçimidir. & $\begin{array}{l}\text { COPRAS- G yöntemi bu } \\
\text { seçimi yapabilir. }\end{array}$ \\
\hline 11. & $\begin{array}{l}\text { (Fouladgar, vd., } \\
\text { 2012) }\end{array}$ & Vaka çalışmasıdır. & $\begin{array}{l}\text { Ağır ekipman yönetimi ve } \\
\text { operasyonunda karar vermenin } \\
\text { önemlidir. }\end{array}$ & $\begin{array}{l}\text { Uygulanabilir bakım } \\
\text { stratejisini değerlendirmek } \\
\text { için COPRAS ve AHP } \\
\text { yöntemleri uygulanabilir. }\end{array}$ \\
\hline 12. & $\begin{array}{l}\text { (Bitarafan, } \\
\text { vd.,2012) }\end{array}$ & Vaka çalışmasıdır. & $\begin{array}{l}\text { İnşaat yöntemlerinin } \\
\text { değerlendirilmesidir. }\end{array}$ & $\begin{array}{l}\text { COPRAS-G yöntemi bu } \\
\text { değerlendirmeyi yapabilir. }\end{array}$ \\
\hline 13. & (Das, vd., 2012) & Vaka çalışmasıdır. & $\begin{array}{l}\text { Hindistan Teknoloji } \\
\text { Enstitüsü'nün performans } \\
\text { değerlendirmesidir. }\end{array}$ & $\begin{array}{l}\text { Fuzzy AHP ve COPRAS } \\
\text { yöntemleri bu değerlendirmeyi } \\
\text { yapabilir. }\end{array}$ \\
\hline 14. & (Barysienè, 2012) & Vaka çalışmasıdır. & $\begin{array}{l}\text { Bir terminalde konteyner } \\
\text { ambalajlama süresinin en aza } \\
\text { indirilmesidir. }\end{array}$ & $\begin{array}{l}\text { Bunun için COPRAS-G } \\
\text { yöntemi kullanılabilir. }\end{array}$ \\
\hline 15. & $\begin{array}{l}\text { (Aghdaie, vd., } \\
\text { 2013) }\end{array}$ & Vaka çalışmasıdır. & $\begin{array}{l}\text { Piyasa segmenti } \\
\text { değerlendirmesi ve seçimidir. }\end{array}$ & $\begin{array}{l}\text { COPRAS-G yöntemi bu } \\
\text { seçimi yapabilir }\end{array}$ \\
\hline 16. & $\begin{array}{l}\text { (Ghorabaee, vd., } \\
\text { 2014) }\end{array}$ & Vaka çalışmasıdır. & Tedarikçi seçimdir. & $\begin{array}{l}\text { Genişletilmiş COPRAS } \\
\text { yöntemi bu seçimi yapabilir. }\end{array}$ \\
\hline 17. & (Madić, vd., 2014) & Vaka çalışmasıdır. & $\begin{array}{l}\text { Tarım ve inşaat sektöründe } \\
\text { faaliyet gösteren bir firma için } \\
\text { tedarikçi performansının } \\
\text { değerlendirmesidir. }\end{array}$ & $\begin{array}{l}\text { COPRAS yöntemi bu } \\
\text { değerlendirmeyi yapabilir. }\end{array}$ \\
\hline 18. & $\begin{array}{l}\text { (Hashemkhani } \\
\text { Zolfani, \& } \\
\text { Bahrami, 2014) }\end{array}$ & Vaka çalışmasıdır. & $\begin{array}{l}\text { Yüksek teknoloji } \\
\text { endüstrilerinin önceliği olan } \\
\text { karar ve politika oluşturma } \\
\text { üzerine odaklanmıştır. }\end{array}$ & $\begin{array}{l}\text { Ölçütleri ağırlıklandırmak için } \\
\text { SWARA ve alternatifleri } \\
\text { sıralamak için COPRAS } \\
\text { yöntemi kullanılabilir. }\end{array}$ \\
\hline 19. & $\begin{array}{l}\text { (Makhesana, } \\
\text { 2015) }\end{array}$ & Vaka çalışmasıdır. & $\begin{array}{l}\text { En iyi üretim sürecinin } \\
\text { seçimidir. }\end{array}$ & $\begin{array}{l}\text { COPRAS yöntemi bu seçimi } \\
\text { yapabilir. }\end{array}$ \\
\hline 20. & (Kouchaksaraei, & Vaka çalışmasıdır. & Sera yatırımlarında yer & SWARA, kriterlerin önem \\
\hline
\end{tabular}




\begin{tabular}{|c|c|c|c|c|}
\hline & vd., 2015) & & seçimidir. & $\begin{array}{l}\text { derecelerinin belirlenmesinde, } \\
\text { COPRAS ise alternatifleri } \\
\text { siralamak için uygulanabilir. }\end{array}$ \\
\hline 21. & $\begin{array}{l}\text { (Vytautas, vd., } \\
\text { 2015) }\end{array}$ & Vaka çalışmasıdır. & $\begin{array}{l}\text { Kentsel alanların yaşam } \\
\text { döngülerinin incelenmesidir. }\end{array}$ & $\begin{array}{l}\text { Bunun için COPRAS yöntemi } \\
\text { kullanılabilir. }\end{array}$ \\
\hline 22. & $\begin{array}{l}\text { (Turanoglu Bekar, } \\
\text { vd., 2016) }\end{array}$ & Vaka çalışmasıdır. & $\begin{array}{l}\text { Modern imalat firmalarında } \\
\text { yeni toplam üretken bakım } \\
\text { stratejisidir. }\end{array}$ & $\begin{array}{l}\text { Önerilen bulanık COPRAS } \\
\text { yöntemi, yeni geliştirilen } \\
\text { performans ölçütlerini } \\
\text { derecelendirmede güven } \\
\text { verici sonuçlar verir. }\end{array}$ \\
\hline 23. & $\begin{array}{l}\text { (Kundakcı \& Işık, } \\
\text { 2016) }\end{array}$ & Vaka çalışmasıdır. & Hava kompresörü seçimidir. & $\begin{array}{l}\text { Alternatiflerin sıralamasını } \\
\text { belirlemek ve en iyisini } \\
\text { seçmek için COPRAS yöntemi } \\
\text { kullanılabilir. }\end{array}$ \\
\hline 24. & $\begin{array}{l}\text { (Organ \& Yalçın, } \\
\text { 2016) }\end{array}$ & Vaka çalışmasıdır. & $\begin{array}{l}\text { Araştırma görevlilerinin } \\
\text { performans değerlendirmesidir. }\end{array}$ & $\begin{array}{l}\text { COPRAS yöntemi bu } \\
\text { değerlendirmeyi yapabilir. }\end{array}$ \\
\hline 25. & $\begin{array}{l}\text { (Adalı \& Iş1k, } \\
\text { 2016) }\end{array}$ & $\begin{array}{l}\text { Vaka çalışması ve } \\
\text { iki yöntemin } \\
\text { karşılaştırılmasıdır. }\end{array}$ & $\begin{array}{l}\text { Firmalar için ofislere en uygun } \\
\text { 1sıtma ve soğutma sistemlerinin } \\
\text { seçimidir. }\end{array}$ & $\begin{array}{l}\text { COPRAS ve ARAS } \\
\text { yöntemleri bu seçimi } \\
\text { yapabilir. }\end{array}$ \\
\hline 26. & $\begin{array}{l}\text { (Bakhouyi, vd., } \\
\text { 2016) }\end{array}$ & Vaka çalışmasıdır. & $\begin{array}{l}\text { Öğrenme Yönetim Sisteminde } \\
\text { zayıf yönlerin tespitidir. }\end{array}$ & $\begin{array}{l}\text { COPRAS yöntemi bu tespiti } \\
\text { yapabilir. }\end{array}$ \\
\hline 27. & $\begin{array}{l}\text { (Sarıçalı \& } \\
\text { Kundakçı, 2016) }\end{array}$ & Vaka çalışmasıdır. & $\begin{array}{l}\text { Tatil için otel alternatifleri } \\
\text { seçimidir. }\end{array}$ & $\begin{array}{l}\text { AHP ve COPRAS yöntemleri } \\
\text { bu seçimi yapabilir. }\end{array}$ \\
\hline 28. & $\begin{array}{l}\text { (Nakhaei, vd., } \\
\text { 2016) }\end{array}$ & Vaka çalışmasıdır. & $\begin{array}{l}\text { Güvenli yer altı alanlarında kriz } \\
\text { anında ulusal elektrik } \\
\text { şebekesini kullanma imkanının } \\
\text { olmadığı koşullarda 1şık } \\
\text { sağlanmasıdır. }\end{array}$ & $\begin{array}{l}\text { Stratejiler arasından en iyi } \\
\text { seçimi COPRAS yöntemi } \\
\text { yapabilir. }\end{array}$ \\
\hline 29. & $\begin{array}{l}\text { (Valipour, vd., } \\
\text { 2017) }\end{array}$ & Vaka çalışmasıdır. & $\begin{array}{l}\text { Derin temel kazı projelerindeki } \\
\text { mevcut risklerin } \\
\text { belirlenmesidir. }\end{array}$ & $\begin{array}{l}\text { COPRAS yöntemi riskleri } \\
\text { belirleyebilir. }\end{array}$ \\
\hline 30. & (Wang, vd., 2017) & Vaka çalışmasıdır. & $\begin{array}{l}\text { Dizel deniz motorlarında sistem } \\
\text { performansı ve güvenliğini } \\
\text { artırmak için hata modu } \\
\text { sıralaması belirlenmesidir. }\end{array}$ & $\begin{array}{l}\text { COPRAS yöntemi bu } \\
\text { siralamay1 yapabilir. }\end{array}$ \\
\hline 31. & $\begin{array}{l}\text { (Yazdani, vd., } \\
\text { 2017) }\end{array}$ & Vaka çalışmasıdır. & $\begin{array}{l}\text { Tasarım konusunda malzeme } \\
\text { değerlendirmesi ve seçimidir. }\end{array}$ & $\begin{array}{l}\text { COPRAS yöntemi bu seçimi } \\
\text { yapabilir. }\end{array}$ \\
\hline 32. & $\begin{array}{l}\text { (Rathi \& } \\
\text { Balamohan, 2017) }\end{array}$ & Vaka çalışmasıdır. & $\begin{array}{l}\text { Uygun bir yangın acil durum } \\
\text { alternatifi seçimidir. }\end{array}$ & $\begin{array}{l}\text { COPRAS yöntemi bu seçimi } \\
\text { yapabilir. }\end{array}$ \\
\hline 33. & $\begin{array}{l}\text { (Amoozad } \\
\text { Mahdiraji, vd., } \\
\text { 2018) }\end{array}$ & Vaka çalışmasıdır. & $\begin{array}{l}\text { Çağdaş mimaride çevresel } \\
\text { sürdürülebilirliğin temel } \\
\text { göstergelerin belirlenmesi ve } \\
\text { öncelik sıralamasının } \\
\text { yapılmasıdır. }\end{array}$ & $\begin{array}{l}\text { COPRAS yöntemi öncelik } \\
\text { sıralamasını yapabilir. }\end{array}$ \\
\hline
\end{tabular}




\begin{tabular}{|c|c|c|c|c|}
\hline 34. & (Zheng, vd., 2018) & Vaka çalışmasıdır. & $\begin{array}{l}\text { KOAH hastalarının ciddiyetinin } \\
\text { değerlendirilmesidir. }\end{array}$ & $\begin{array}{l}\text { HFL-COPRAS yöntemi bu } \\
\text { değerlendirmeyi yapabilir. }\end{array}$ \\
\hline 35. & (Garg, vd., 2018) & Vaka çalışmasıdır. & $\begin{array}{l}\text { E-öğrenme sistemlerini etkili } \\
\text { bir şekilde analiz etme ve } \\
\text { yönetme konusunda eğitim ve } \\
\text { eğitim organizasyonlarındaki } \\
\text { liderlere karar desteği } \\
\text { sağlamasıdır. }\end{array}$ & $\begin{array}{l}\text { Fuzzy COPRAS yöntemi bu } \\
\text { desteği sağlayabilir. }\end{array}$ \\
\hline 36. & (Mishra, vd., 2019) & Vaka çalışmasıdır. & $\begin{array}{l}\text { Araç sigortası hizmetleri } \\
\text { kalitesi } \\
\text { seçim problemleridir. }\end{array}$ & $\begin{array}{l}\text { Hizmet kalitesinin } \\
\text { değerlendirilmesinde Shapley } \\
\text { COPRAS yöntemi } \\
\text { kullanılabilir. }\end{array}$ \\
\hline 37. & $\begin{array}{l}\text { (Kumari \& Mishra, } \\
\text { 2020) }\end{array}$ & Vaka çalışmasıdır. & Yeşil tedarikçi seçimidir. & $\begin{array}{l}\text { IF-COPRAS yöntemi bu } \\
\text { seçimi yapabilir. }\end{array}$ \\
\hline 38. & $\begin{array}{l}\text { (Darko \& Liang, } \\
\text { 2020) }\end{array}$ & Vaka çalışmasıdır. & $\begin{array}{l}\text { Mobil ödeme platformu } \\
\text { seçimidir. }\end{array}$ & $\begin{array}{l}\text { WDHFMSM - COPRAS } \\
\text { yöntemi bu seçimi yapabilir. }\end{array}$ \\
\hline 39. & $\begin{array}{l}\text { (Roozbahani, vd., } \\
\text { 2020) }\end{array}$ & Vaka çalışmasıdır. & $\begin{array}{l}\text { Havzalar arası su transferi } \\
\text { konusunda senaryo seçimidir. }\end{array}$ & $\begin{array}{l}\text { COPRAS yöntemi bu seçimi } \\
\text { yapabilir. }\end{array}$ \\
\hline 40. & $\begin{array}{l}\text { (Narayanamoorthy, } \\
\text { vd., 2020) }\end{array}$ & Vaka çalışmasıdır. & $\begin{array}{l}\text { Sürdürülebilir yakıt } \\
\text { kaynaklarının seçimdir. }\end{array}$ & $\begin{array}{l}\text { Fuzzy COPRAS yöntemi bu } \\
\text { seçimi yapabilir. }\end{array}$ \\
\hline 41. & $\begin{array}{l}\text { (Sałabun, vd., } \\
\text { 2020) }\end{array}$ & $\begin{array}{l}\text { Yöntemlerin } \\
\text { karşılaştırılması ve } \\
\text { vaka çalışmasıdır. }\end{array}$ & $\begin{array}{l}\text { Çok Kriterli Karar Analizi } \\
\text { (MCDA) yöntemlerini } \\
\text { karşılaştırılmasıdır. }\end{array}$ & $\begin{array}{l}\text { Farklı MCDA yöntemleriyle } \\
\text { elde edilen sıralamalar } \\
\text { birbirleriyle benzerdir. }\end{array}$ \\
\hline 42. & $\begin{array}{l}\text { (Kustiyahningsih, } \\
\text { \& Aini, 2020) }\end{array}$ & Vaka çalışmasıdır. & Yeni öğrencilerin seçimidir. & $\begin{array}{l}\text { FAHP ve COPRAS } \\
\text { yöntemleri bu seçimi } \\
\text { yapabilir. }\end{array}$ \\
\hline 43. & $\begin{array}{l}\text { (Dhiman \& Deb, } \\
\text { 2020) }\end{array}$ & Vaka çalışmasıdır. & $\begin{array}{l}\text { Hibrit rüzgar çiftlikleri } \\
\text { seçimdir. }\end{array}$ & $\begin{array}{l}\text { TOPSIS ve COPRAS } \\
\text { yöntemleri bu seçimi } \\
\text { yapabilir. }\end{array}$ \\
\hline 44. & (Mishra, vd., 2020) & Vaka çalışmasıdır. & $\begin{array}{l}\text { Optimal bir biyoenerji üretim } \\
\text { teknolojisi seçimidir. }\end{array}$ & $\begin{array}{l}\text { SWARA ve COPRAS } \\
\text { yöntemleri bu seçimi } \\
\text { yapabilir. }\end{array}$ \\
\hline 45. & $\begin{array}{l}\text { (Mercangoz, vd., } \\
2020 \text { ) }\end{array}$ & Vaka çalışmasıdır. & $\begin{array}{l}\text { Lojistik performans } \\
\text { değerlendirilmesidir. }\end{array}$ & $\begin{array}{l}\text { COPRAS-G yöntemi bu } \\
\text { değerlendirmeyi yapabilir. }\end{array}$ \\
\hline 46. & $\begin{array}{l}\text { (Nweze \& Achebo, } \\
\text { 2021) }\end{array}$ & Vaka çalışmasıdır. & $\begin{array}{l}\text { Optimum proses } \\
\text { parametrelerinin seçimidir. }\end{array}$ & $\begin{array}{l}\text { COPRAS-ARAS yöntemi bu } \\
\text { seçimi yapabilir. }\end{array}$ \\
\hline 47. & $\begin{array}{l}\text { (Kablan \& } \\
\text { Erdoğan, 2021) }\end{array}$ & Vaka çalışmasıdır. & $\begin{array}{l}\text { Kamu, Özel ve Yabancı } \\
\text { Sermayeli bankaların finansal } \\
\text { performansıdır. }\end{array}$ & $\begin{array}{l}\text { COPRAS yöntemi bu } \\
\text { karşılaştırmayı yapabilir. }\end{array}$ \\
\hline
\end{tabular}




\section{3. Çalışmanın Amacı ve Kapsamı}

Çalışmanın amacı karar verme sürecinde COPRAS yöntemi uygulamasıdır. Bunun için bir vaka çalışması yapılmıştır. Uygulamanın gösterimi için Batı Avrupa: Şehre Göre Mevcut Yaşam Kalitesi İndeksi kullanılmıştır. Yaşam Kalitesi İndeksi sekiz kriterden oluşmaktadır. Bu kriterler Satın Alma Gücü İndeksi, Güvenlik İndeksi, Sağlık İndeksi, İklim İndeksi, Yaşam Maliyeti İndeksi, Emlak Fiyatı Gelir Oranı, Trafik İşe Gidip Gelme Süresi İndeksi ve Kirlilik İndeksidir. Yaşam Kalitesi İndeksi hesaplamasında kullanılan bu kriterler daha yüksek daha iyidir ve daha düşük daha iyidir şeklinde sınıflandırılır.

Uygulama sürecinde kriterlerin yönü Satın Alma Gücü İndeksi (C1), Güvenlik İndeksi (C2), Sağlık İndeksi (C3) ve İklim İndeksi (C4) için maksimum olarak belirlenmiştir. Diğer kriterler; Yaşam Maliyeti İndeksi (C5), Emlak Fiyatı Gelir Oranı (C6), Trafik İşe Gidip Gelme Süresi İndeksi (C7) ve Kirlilik İndeksi(C8) için minimum olarak belirlenmiştir. Amaç, en yüksek Yaşam Kalitesi İndeksine sahip şehir ve şehirlerin seçimidir.

\section{Copras Yöntemi}

COPRAS Yönteminin matematiksel gösterimi için (Wang, vd., 2017, s. 1007) çalışmasından yararlanılmıştır. Yöntem, karar matrisi oluşturulduktan sonra beş aşamada gerçekleşir.

$\mathrm{M}$ alternatiflerin $\mathrm{n}$ kriterlere göre performans derecelendirmelerinin bir karar matrisi $X=\left[x_{i j}\right]_{m \times n}$ olarak temsil edildiği varsayılmıştır. Burada $\mathrm{x}_{\mathrm{ij}}$, j. kriterdeki i. alternatifin derecelendirmesidir.

Aşama 1. Normalleştirilmiş karar matrisi $R=\left[r_{i j}\right]_{m \times n}$ elde edilir.

$$
r_{i j}=\frac{x_{i j}}{\sum_{i=1}^{m} x_{i j}}, i=1,2, \ldots m, j=1,2, \ldots n .
$$

Aşama 2. Ağırlıklı normalleştirilmiş karar matrisi $R^{\prime}=\left[r_{i j}^{\prime}\right]_{m \times n}$ hesaplanır.

$$
r_{i j}^{\prime}=w_{j} r_{i j}, i=1,2, \ldots m, j=1,2, \ldots n,
$$

burada $\mathrm{w}_{\mathrm{j}}, \mathrm{j}$. kriterin ağırlığgdır ve $\sum_{j=1}^{n} w_{j}$ gösterilir.

Aşama 3. Aşağıdaki formüller kullanılarak fayda ve maliyet kriterleri için ağırlıklı normalleştirilmiş değerlerin toplamı hesaplanır.

$$
\begin{aligned}
& P_{i}=\sum_{j=1}^{k} r_{i j}^{\prime}, \\
& R_{i}=\sum_{j=1}^{n-k} r_{i j}^{\prime},
\end{aligned}
$$

Aşama 4. Alternatiflerin göreceli önemleri belirlenir.

$$
Q_{i}=P_{i}+\frac{\sum_{i=1}^{m} R_{i}}{R_{i} \sum_{i=1}^{m} 1 / R_{i}}, i=1,2, \ldots, m .
$$


Aşama 5. Tüm alternatiflerin önceliklerini en verimli olanla karşılaştırarak her alternatif için fayda derecesi hesaplanır.

$$
N_{i}=\frac{Q_{j}}{Q_{\max }} \times 100 \%, i=1,2, \ldots, m,
$$

burada Q max maksimum göreli anlamlılık değeridir.

\section{Copras Yönteminin Çözümü}

COPRAS yönteminin çözümü için (Ayçin, 2020, s. 67-71) çalışmasından yararlanılmıştır.

Aşama 1. Karar matrisinin oluşturulmasıdır.

İlk aşamada, karar matrisi oluşturulur. Tablo 2 karar matrisini göstermektedir.

Tablo 2: Karar Matrisi

\begin{tabular}{|c|l|c|c|c|c|c|c|c|c|}
\hline \multicolumn{1}{|c|}{ B } & C & D & E & F & G & H & I & J \\
\hline 1 & Kriter Yönü & maks & maks & maks & maks & min & min & min & min \\
\hline 3 & Kentler/ Kriterler & C1 & C2 & C3 & C4 & C5 & C6 & C7 & C8 \\
\hline 4 & Zürih, İsviçre & 113,62 & 83,65 & 75,34 & 81,48 & 130,32 & 8,25 & 33,78 & 17,31 \\
\hline 5 & Lahey (Den Haag), Hollanda & 89,57 & 74,33 & 76,80 & 90,61 & 71,11 & 5,90 & 29,86 & 21,18 \\
\hline 6 & Eindhoven, Hollanda & 82,14 & 75,95 & 73,04 & 85,38 & 79,73 & 6,64 & 25,57 & 22,55 \\
\hline 7 & Cenevre, İsviçre & 111,38 & 72,82 & 72,60 & 82,61 & 123,97 & 8,89 & 25,49 & 26,44 \\
\hline 8 & Viyana, Avusturya & 82,64 & 74,56 & 79,06 & 81,77 & 70,36 & 13,36 & 26,45 & 17,55 \\
\hline 9 & Lüksemburg, Lüksemburg & 99,46 & 71,85 & 74,85 & 82,62 & 87,66 & 12,05 & 33,15 & 20,75 \\
\hline 10 & Münih, Almanya & 89,86 & 82,58 & 78,15 & 75,98 & 81,31 & 16,59 & 33,01 & 24,64 \\
\hline 11 & Düsseldorf, Almanya & 92,78 & 68,50 & 72,33 & 86,37 & 69,69 & 9,11 & 30,31 & 33,68 \\
\hline 12 & Frankfurt, Almanya & 100,14 & 58,35 & 74,37 & 84,72 & 74,01 & 9,78 & 25,10 & 37,78 \\
\hline 13 & Rotterdam, Hollanda & 86,82 & 64,13 & 76,88 & 87,89 & 76,77 & 6,29 & 30,03 & 42,50 \\
\hline 14 & Amsterdam, Hollanda & 79,65 & 66,49 & 69,26 & 87,45 & 82,55 & 10,61 & 29,41 & 30,68 \\
\hline 15 & Stuttgart, Almanya & 95,43 & 71,60 & 72,20 & 81,07 & 71,30 & 9,86 & 34,80 & 44,79 \\
\hline 16 & Hamburg, Almanya & 85,04 & 56,76 & 74,68 & 82,75 & 73,56 & 11,54 & 34,21 & 31,09 \\
\hline 17 & Berlin, Almanya & 85,90 & 58,68 & 69,50 & 83,35 & 70,39 & 10,44 & 34,12 & 39,40 \\
\hline 18 & Cologne, Almanya & 86,17 & 56,18 & 74,83 & 84,85 & 72,46 & 10,12 & 36,39 & 41,47 \\
\hline 19 & Antwerp, Belçika & 77,83 & 62,79 & 80,53 & 85,42 & 76,68 & 6,01 & 37,15 & 59,95 \\
\hline 20 & Lyon, Fransa & 73,47 & 54,00 & 78,08 & 88,51 & 80,34 & 9,32 & 33,78 & 51,09 \\
\hline 21 & Brüksel, Belçika & 81,24 & 49,41 & 74,94 & 83,85 & 77,56 & 6,42 & 37,11 & 62,13 \\
\hline 22 & Paris, Fransa & 69,48 & 45,86 & 79,68 & 88,39 & 91,77 & 21,47 & 40,87 & 64,64 \\
\hline
\end{tabular}

Aşama 2. Karar matrisinin normalize edilmesidir.

Bunun içinC27 hücresine $=$ C4/TOPLA(\$C\$4:\$C\$22) formülü yazılır ve C13-C45 aralığına kopyalanır. Aynı işlem C1- C8 aralığındaki tüm hücreler için yapılır. Tablo 3 normalize edilmiş karar matrisini göstermektedir. 
Tablo 3: Normalize Karar Matrisi

\begin{tabular}{|c|l|c|c|c|c|c|c|c|c|}
\hline \multicolumn{1}{|c|}{ B } & C & D & E & F & G & H & 1 & J \\
\hline 24 & Kriter Yönü & maks & maks & maks & maks & min & min & min & min \\
\hline 26 & Kentler/ Kriterler & C1 & C2 & C3 & C4 & C5 & C6 & C7 & C8 \\
\hline 27 & Zürih, İsviçre & 0,0675 & 0,0670 & 0,0528 & 0,0508 & 0,0835 & 0,0428 & 0,0553 & 0,0251 \\
\hline 28 & Lahey (Den Haag), Hollanda & 0,0532 & 0,0595 & 0,0538 & 0,0565 & 0,0455 & 0,0306 & 0,0489 & 0,0307 \\
\hline 29 & Eindhoven, Hollanda & 0,0488 & 0,0608 & 0,0512 & 0,0532 & 0,0511 & 0,0345 & 0,0419 & 0,0327 \\
\hline 30 & Cenevre, İsviçre & 0,0662 & 0,0583 & 0,0509 & 0,0515 & 0,0794 & 0,0461 & 0,0417 & 0,0383 \\
\hline 31 & Viyana, Avusturya & 0,0491 & 0,0597 & 0,0554 & 0,0509 & 0,0451 & 0,0693 & 0,0433 & 0,0254 \\
\hline 32 & Lüksemburg, Lüksemburg & 0,0591 & 0,0575 & 0,0524 & 0,0515 & 0,0561 & 0,0625 & 0,0543 & 0,0301 \\
\hline 33 & Münih, Almanya & 0,0534 & 0,0661 & 0,0548 & 0,0473 & 0,0521 & 0,0861 & 0,0541 & 0,0357 \\
\hline 34 & Düsseldorf, Almanya & 0,0551 & 0,0549 & 0,0507 & 0,0538 & 0,0446 & 0,0473 & 0,0496 & 0,0488 \\
\hline 35 & Frankfurt, Almanya & 0,0595 & 0,0467 & 0,0521 & 0,0528 & 0,0474 & 0,0508 & 0,0411 & 0,0548 \\
\hline 36 & Rotterdam, Hollanda & 0,0516 & 0,0514 & 0,0539 & 0,0548 & 0,0492 & 0,0326 & 0,0492 & 0,0616 \\
\hline 37 & Amsterdam, Hollanda & 0,0473 & 0,0533 & 0,0485 & 0,0545 & 0,0529 & 0,0551 & 0,0482 & 0,0445 \\
\hline 38 & Stuttgart, Almanya & 0,0567 & 0,0573 & 0,0506 & 0,0505 & 0,0457 & 0,0512 & 0,0570 & 0,0649 \\
\hline 39 & Hamburg, Almanya & 0,0505 & 0,0455 & 0,0523 & 0,0516 & 0,0471 & 0,0599 & 0,0560 & 0,0451 \\
\hline 40 & Berlin, Almanya & 0,0511 & 0,0470 & 0,0487 & 0,0519 & 0,0451 & 0,0542 & 0,0559 & 0,0571 \\
\hline 41 & Cologne, Almanya & 0,0512 & 0,0450 & 0,0524 & 0,0529 & 0,0464 & 0,0525 & 0,0596 & 0,0601 \\
\hline 42 & Antwerp, Belçika & 0,0463 & 0,0503 & 0,0564 & 0,0532 & 0,0491 & 0,0312 & 0,0608 & 0,0869 \\
\hline 43 & Lyon, Fransa & 0,0437 & 0,0433 & 0,0547 & 0,0551 & 0,0514 & 0,0484 & 0,0553 & 0,0741 \\
\hline 44 & Brüksel, Belçika & 0,0483 & 0,0396 & 0,0525 & 0,0522 & 0,0497 & 0,0333 & 0,0608 & 0,0901 \\
\hline 45 & Paris, Fransa & 0,0413 & 0,0367 & 0,0558 & 0,0551 & 0,0588 & 0,1114 & 0,0669 & 0,0937 \\
\hline
\end{tabular}

Aşama 3. Normalize edilen karar matrisinin ağırlıklandırılmasıdır.

Bunun için C53 hücresine $=\mathrm{C} 27 * \$ C \$ 48$ formülü yazılır ve C53-C71 aralığına kopyalanır. Aynı işlem C1- C8 aralığındaki tüm hücreler için yapılır. Tablo 4 kriter ve ağılırlıkları, Tablo 5 ağırlıklandırılmış normalize karar matrisini göstermektedir.

Tablo 4: Kriterler ve Ağırlıkları

\begin{tabular}{|c|l|c|c|c|c|c|c|c|c|}
\hline \multicolumn{1}{|c|}{ B } & C & D & E & F & G & H & I & J \\
\hline 46 & Kriter & C1 & C2 & C3 & C4 & C5 & C6 & C7 & C8 \\
\hline 48 & Kriter Ağırlıkları & 0,125 & 0,125 & 0,125 & 0,125 & 0,125 & 0,125 & 0,125 & 0,125 \\
\hline
\end{tabular}


Tablo 5: A ğırlıklandırılmış Normalize Karar Matrisi

\begin{tabular}{|c|l|c|c|c|c|c|c|c|c|}
\hline \multicolumn{1}{|c|}{ B } & C & D & E & F & G & H & I & J \\
\hline 50 & Kriter Yönü & maks & maks & maks & maks & min & min & min & min \\
\hline 52 & Kentler/ Kriterler & C1 & C2 & C3 & C4 & C5 & C6 & C7 & C8 \\
\hline 53 & Zürih, İsviçre & 0,0084 & 0,0084 & 0,0066 & 0,0063 & 0,0104 & 0,0054 & 0,0069 & 0,0031 \\
\hline 54 & Lahey (Den Haag), Hollanda & 0,0067 & 0,0074 & 0,0067 & 0,0071 & 0,0057 & 0,0038 & 0,0061 & 0,0038 \\
\hline 55 & Eindhoven, Hollanda & 0,0061 & 0,0076 & 0,0064 & 0,0066 & 0,0064 & 0,0043 & 0,0052 & 0,0041 \\
\hline 56 & Cenevre, İsviçre & 0,0083 & 0,0073 & 0,0064 & 0,0064 & 0,0099 & 0,0058 & 0,0052 & 0,0048 \\
\hline 57 & Viyana, Avusturya & 0,0061 & 0,0075 & 0,0069 & 0,0064 & 0,0056 & 0,0087 & 0,0054 & 0,0032 \\
\hline 58 & Lüksemburg, Lüksemburg & 0,0074 & 0,0072 & 0,0066 & 0,0064 & 0,0070 & 0,0078 & 0,0068 & 0,0038 \\
\hline 59 & Münih, Almanya & 0,0067 & 0,0083 & 0,0068 & 0,0059 & 0,0065 & 0,0108 & 0,0068 & 0,0045 \\
\hline 60 & Düsseldorf, Almanya & 0,0069 & 0,0069 & 0,0063 & 0,0067 & 0,0056 & 0,0059 & 0,0062 & 0,0061 \\
\hline 61 & Frankfurt, Almanya & 0,0074 & 0,0058 & 0,0065 & 0,0066 & 0,0059 & 0,0063 & 0,0051 & 0,0068 \\
\hline 62 & Rotterdam, Hollanda & 0,0064 & 0,0064 & 0,0067 & 0,0068 & 0,0061 & 0,0041 & 0,0061 & 0,0077 \\
\hline 63 & Amsterdam, Hollanda & 0,0059 & 0,0067 & 0,0061 & 0,0068 & 0,0066 & 0,0069 & 0,0060 & 0,0056 \\
\hline 64 & Stuttgart, Almanya & 0,0071 & 0,0072 & 0,0063 & 0,0063 & 0,0057 & 0,0064 & 0,0071 & 0,0081 \\
\hline 65 & Hamburg, Almanya & 0,0063 & 0,0057 & 0,0065 & 0,0064 & 0,0059 & 0,0075 & 0,0070 & 0,0056 \\
\hline 66 & Berlin, Almanya & 0,0064 & 0,0059 & 0,0061 & 0,0065 & 0,0056 & 0,0068 & 0,0070 & 0,0071 \\
\hline 67 & Cologne, Almanya & 0,0064 & 0,0056 & 0,0066 & 0,0066 & 0,0058 & 0,0066 & 0,0074 & 0,0075 \\
\hline 68 & Antwerp, Belçika & 0,0058 & 0,0063 & 0,0071 & 0,0067 & 0,0061 & 0,0039 & 0,0076 & 0,0109 \\
\hline 69 & Lyon, Fransa & 0,0055 & 0,0054 & 0,0068 & 0,0069 & 0,0064 & 0,0060 & 0,0069 & 0,0093 \\
\hline 70 & Brüksel, Belçika & 0,0060 & 0,0049 & 0,0066 & 0,0065 & 0,0062 & 0,0042 & 0,0076 & 0,0113 \\
\hline 71 & Paris, Fransa & 0,0052 & 0,0046 & 0,0070 & 0,0069 & 0,0073 & 0,0139 & 0,0084 & 0,0117 \\
\hline
\end{tabular}

Aşama 4. Ağırlıklandırılmış normalize indekslerinin toplanmasıdır.

Bunun için ilk olarak, C76 hücresine $=$ TOPLA(C53:F53) formülü yazılır. Daha sonra, D76 hücresine $=$ TOPLA(G53:J53) formülü yazılır. Tablo 6 indekslerin toplamını göstermektedir.

Tablo 6: İndekslerin Toplamı

\begin{tabular}{|c|l|c|c|}
\hline 74 & \multicolumn{1}{|c|}{ B } & C & D \\
\hline 75 & Kentler/ Kriterler & S+i & S-i \\
\hline 76 & Zürih, İsviçre & 0,0298 & 0,0258 \\
\hline 77 & Lahey (Den Haag), Hollanda & 0,0279 & 0,0195 \\
\hline 78 & Eindhoven, Hollanda & 0,0268 & 0,0200 \\
\hline 79 & Cenevre, İsviçre & 0,0284 & 0,0257 \\
\hline 80 & Viyana, Avusturya & 0,0269 & 0,0229 \\
\hline 81 & Lüksemburg, Lüksemburg & 0,0276 & 0,0254 \\
\hline 82 & Münih, Almanya & 0,0277 & 0,0285 \\
\hline 83 & Düsseldorf, Almanya & 0,0268 & 0,0238 \\
\hline
\end{tabular}




\begin{tabular}{|c|l|c|c|}
\hline 84 & Frankfurt, Almanya & 0,0264 & 0,0243 \\
\hline 85 & Rotterdam, Hollanda & 0,0264 & 0,0241 \\
\hline 86 & Amsterdam, Hollanda & 0,0255 & 0,0251 \\
\hline 87 & Stuttgart, Almanya & 0,0269 & 0,0273 \\
\hline 88 & Hamburg, Almanya & 0,0250 & 0,0260 \\
\hline 89 & Berlin, Almanya & 0,0248 & 0,0265 \\
\hline 90 & Cologne, Almanya & 0,0252 & 0,0273 \\
\hline 91 & Antwerp, Belçika & 0,0258 & 0,0285 \\
\hline 92 & Lyon, Fransa & 0,0246 & 0,0287 \\
\hline 93 & Brüksel, Belçika & 0,0241 & 0,0292 \\
\hline 94 & Paris, Fransa & 0,0236 & 0,0414 \\
\hline
\end{tabular}

Aşama 5. Karar alternatiflerinin göreli önem düzeylerinin hesaplanmasıdır.

Bunun için ilk olarak, E99 hücresine =MIN(D76:D94) formülü yazılır. İkinci olarak, F99 hücresine =TOPLA(D76:D94) formülü yazılır. Üçüncü olarak, G99 hücresine =\$E\$99/D76 formülü yazılır. Dördüncü olarak, H99 hücresine =TOPLA(G99:G117) formülü yazılır. Son olarak, I99 hücresine $=\mathrm{C} 99+(\$ E \$ 99 * \$ F \$ 99) /(\mathrm{D} 99 * \$ H \$ 99)$ formülü yazılır. Tablo 7 karar alternatiflerinin göreli önem düzeylerini göstermektedir.

Tablo 7: Önem Düzeyleri

\begin{tabular}{|c|c|c|c|c|c|c|c|c|}
\hline 97 & B & $\mathrm{C}$ & $\mathrm{D}$ & $\mathrm{E}$ & $\mathrm{F}$ & $\mathrm{G}$ & $\mathrm{H}$ & I \\
\hline 98 & Kentler/ Kriterler & $\mathbf{S}+\mathbf{i}$ & S-i & S-min & $\Sigma$ S-i & S-min/S-i & $\Sigma S-\min / \mathbf{S}-\mathbf{i}$ & Qi \\
\hline 99 & Zürih, İsviçre & 0,0298 & 0,0258 & 0,0195 & 0,5000 & 0,7536 & 14,3977 & 0,0559 \\
\hline 100 & Lahey (Den Haag), Hollanda & 0,0279 & 0,0195 & & & 1,0000 & & 0,0626 \\
\hline 101 & Eindhoven, Hollanda & 0,0268 & 0,0200 & & & 0,9730 & & 0,0605 \\
\hline 102 & Cenevre, İsviçre & 0,0284 & 0,0257 & & & 0,7576 & & 0,0547 \\
\hline 103 & Viyana, Avusturya & 0,0269 & 0,0229 & & & 0,8504 & & 0,0564 \\
\hline 104 & Lüksemburg, Lüksemburg & 0,0276 & 0,0254 & & & 0,7671 & & 0,0542 \\
\hline 105 & Münih, Almanya & 0,0277 & 0,0285 & & & 0,6833 & & 0,0514 \\
\hline 106 & Düsseldorf, Almanya & 0,0268 & 0,0238 & & & 0,8182 & & 0,0552 \\
\hline 107 & Frankfurt, Almanya & 0,0264 & 0,0243 & & & 0,8028 & & 0,0543 \\
\hline 108 & Rotterdam, Hollanda & 0,0264 & 0,0241 & & & 0,8087 & & 0,0545 \\
\hline 109 & Amsterdam, Hollanda & 0,0255 & 0,0251 & & & 0,7766 & & 0,0524 \\
\hline 110 & Stuttgart, Almanya & 0,0269 & 0,0273 & & & 0,7120 & & 0,0516 \\
\hline 111 & Hamburg, Almanya & 0,0250 & 0,0260 & & & 0,7485 & & 0,0510 \\
\hline 112 & Berlin, Almanya & 0,0248 & 0,0265 & & & 0,7338 & & 0,0503 \\
\hline 113 & Cologne, Almanya & 0,0252 & 0,0273 & & & 0,7124 & & 0,0499 \\
\hline 114 & Antwerp, Belçika & 0,0258 & 0,0285 & & & 0,6830 & & 0,0495 \\
\hline 115 & Lyon, Fransa & 0,0246 & 0,0287 & & & 0,6796 & & 0,0482 \\
\hline 116 & Brüksel, Belçika & 0,0241 & 0,0292 & & & 0,6661 & & 0,0472 \\
\hline
\end{tabular}


$117 \quad$ Paris, Fransa

\begin{tabular}{|l|l|}
0,0236 & 0,0414 \\
\hline
\end{tabular}

0,4708

0,0400

Aşama 6. Karar alternatiflerinin performans indekslerinin hesaplanmasıdır.

Bunun için ilk olarak, J122 hücresine =I122/MAK(\$I $\$ 122: \$ I$ I40)*100 formülü yazılır. Daha sonra, K122 hücresine $=$ RANK $(J 122 ; \$ J 122: \$ J \$ 140 ; 0)$ formülü yazılır. Tablo 8 performans indeks değerlerini göstermektedir.

Tablo 8: Performans İndeks Değerleri

\begin{tabular}{|l|l|c|c|c|c|c|c|c|c|c|}
\hline 120 & & C & D & E & F & G & H & I & J & K \\
\hline 121 & Kentler/ Kriterler & S+i & S-i & S-min & IS-i & $\begin{array}{c}\text { S-min / } \\
\text { S-i }\end{array}$ & $\begin{array}{c}\text { IS-min / } \\
\text { S-i }\end{array}$ & Qi & Pi & Siralama \\
\hline 122 & Zürih, İsviçre & $\mathbf{0 , 0 3}$ & $\mathbf{0 , 0 3}$ & $\mathbf{0 , 0 2}$ & $\mathbf{0 , 5 0}$ & $\mathbf{0 , 7 5}$ & $\mathbf{1 4 , 4 0}$ & $\mathbf{0 , 0 6}$ & $\mathbf{8 9 , 3 4}$ & $\mathbf{4}$ \\
\hline 123 & Lahey (Den Haag), Hollanda & $\mathbf{0 , 0 3}$ & $\mathbf{0 , 0 2}$ & & & $\mathbf{1 , 0 0}$ & & $\mathbf{0 , 0 6}$ & $\mathbf{1 0 0 , 0 0}$ & $\mathbf{1}$ \\
\hline 124 & Eindhoven, Hollanda & $\mathbf{0 , 0 3}$ & $\mathbf{0 , 0 2}$ & & & $\mathbf{0 , 9 7}$ & & $\mathbf{0 , 0 6}$ & $\mathbf{9 6 , 7 0}$ & $\mathbf{2}$ \\
\hline 125 & Cenevre, İsviçre & 0,03 & 0,03 & & & 0,76 & & 0,05 & 87,32 & 6 \\
\hline 126 & Viyana, Avusturya & $\mathbf{0 , 0 3}$ & $\mathbf{0 , 0 2}$ & & & $\mathbf{0 , 8 5}$ & & $\mathbf{0 , 0 6}$ & $\mathbf{9 0 , 1 4}$ & $\mathbf{3}$ \\
\hline 127 & Lüksemburg, Lüksemburg & 0,03 & 0,03 & & & 0,77 & & 0,05 & 86,59 & 9 \\
\hline 128 & Münih, Almanya & 0,03 & 0,03 & & & 0,68 & & 0,05 & 82,16 & 12 \\
\hline 129 & Düsseldorf, Almanya & $\mathbf{0 , 0 3}$ & $\mathbf{0 , 0 2}$ & & & $\mathbf{0 , 8 2}$ & & $\mathbf{0 , 0 6}$ & $\mathbf{8 8 , 2 1}$ & $\mathbf{5}$ \\
\hline 130 & Frankfurt, Almanya & 0,03 & 0,02 & & & 0,80 & & 0,05 & 86,69 & 8 \\
\hline 131 & Rotterdam, Hollanda & 0,03 & 0,02 & & & 0,81 & & 0,05 & 87,11 & 7 \\
\hline 132 & Amsterdam, Hollanda & 0,03 & 0,03 & & & 0,78 & & 0,05 & 83,73 & 10 \\
\hline 133 & Stuttgart, Almanya & 0,03 & 0,03 & & & 0,71 & & 0,05 & 82,45 & 11 \\
\hline 134 & Hamburg, Almanya & 0,02 & 0,03 & & & 0,75 & & 0,05 & 81,43 & 13 \\
\hline 135 & Berlin, Almanya & 0,02 & 0,03 & & & 0,73 & & 0,05 & 80,37 & 14 \\
\hline 136 & Cologne, Almanya & $\mathbf{0 , 0 3}$ & $\mathbf{0 , 0 3}$ & & & $\mathbf{0 , 7 1}$ & & $\mathbf{0 , 0 5}$ & $\mathbf{7 9 , 7 5}$ & $\mathbf{1 5}$ \\
\hline 138 & Antwerp, Belçika & $\mathbf{0 , 0 3}$ & $\mathbf{0 , 0 3}$ & & & $\mathbf{0 , 6 8}$ & & $\mathbf{0 , 0 5}$ & $\mathbf{7 9 , 0 5}$ & $\mathbf{1 6}$ \\
\hline 139 & Lyon, Fransa & $\mathbf{0 , 0 2}$ & $\mathbf{0 , 0 3}$ & & & $\mathbf{0 , 6 8}$ & & $\mathbf{0 , 0 5}$ & $\mathbf{7 6 , 9 8}$ & $\mathbf{1 7}$ \\
\hline 140 & Brüksel, Belçika & $\mathbf{0 , 0 2}$ & $\mathbf{0 , 0 3}$ & & & $\mathbf{0 , 6 7}$ & & $\mathbf{0 , 0 5}$ & $\mathbf{7 5 , 4 0}$ & $\mathbf{1 8}$ \\
\hline 141 & Paris, Fransa & $\mathbf{0 , 0 2}$ & $\mathbf{0 , 0 4}$ & & & $\mathbf{0 , 4 7}$ & & $\mathbf{0 , 0 4}$ & $\mathbf{6 3 , 8 4}$ & $\mathbf{1 9}$ \\
\hline
\end{tabular}

Batı Avrupa şehre göre mevcut yaşam kalitesi değerlendirilmesine yönelik COPRAS yöntemi sonuçlarına göre, en iyi yaşam kalitesine sahip ilk beş şehir sırasıyla Lahey (Den Haag), Eindhoven, Viyana, Zürih, ve Düsseldorf şehirleridir. Son beş şehir sırasıyla Cologne, Antwerp, Lyon, Brüksel ve Paris şehirleridir. Sonuçlar Yaşam Kalitesi İndeksi (YKİ) siralamasına göre tutarlıdır.

\section{Duyarlılık Analizi}

Bu bölümde duyarlılık analizi yapılmıştır. Bunun için parametrik olmayan serilerin korelasyon ilişkisi Kendall's tau_b ve Spearman's rho yaklaşımlarıyla incelenmiştir. Tablo 9 Yaşam Kalitesi İndeksi ile COPRAS yöntemi performans sonuçları arasındaki ilişkiyi göstermektedir. 
Tablo 9: Parametrik Olmayan Korelasyonlar

\begin{tabular}{|c|c|c|c|c|}
\hline \multicolumn{5}{|c|}{ Korelasyonlar } \\
\hline & & & YKE & $\mathrm{Pi}$ \\
\hline \multirow[t]{6}{*}{ Kendall's tau_b } & \multirow[t]{3}{*}{ YKI } & Korelasyon katsayısı & 1.000 & $.836^{* *}$ \\
\hline & & Sig. (2 kuyruklu) & . & .000 \\
\hline & & $\mathrm{N}$ & 19 & 19 \\
\hline & \multirow[t]{3}{*}{$\mathrm{Pi}$} & Korelasyon katsayısı & $.836^{* *}$ & 1.000 \\
\hline & & Sig. (2 kuyruklu) & .000 & . \\
\hline & & $\mathrm{N}$ & 19 & 19 \\
\hline \multirow[t]{6}{*}{ Spearman's rho } & \multirow[t]{3}{*}{ YKI } & Korelasyon katsayısı & 1.000 & $.935^{* *}$ \\
\hline & & Sig. (2 kuyruklu) & . & .000 \\
\hline & & $\mathrm{N}$ & 19 & 19 \\
\hline & \multirow[t]{3}{*}{$\mathrm{Pi}$} & Korelasyon katsayısı & $.935^{* *}$ & 1.000 \\
\hline & & Sig. (2 kuyruklu) & .000 & . \\
\hline & & $\mathrm{N}$ & 19 & 19 \\
\hline
\end{tabular}

Tablo 9'a göre, Yaşam Kalitesi İndeksi ile COPRAS yöntemi performans sonuçları arasında güçlü pozitif yönlü bir ilişki vardır. Korelasyonlar istatistiksel olarak anlamlıdır. Bu sonuç, COPRAS yöntemiyle yapılan çalışmaların güvenilirliğini konusunda önemli bir kanıt sunmaktadir.

\section{Sonuç}

Çok kriterli karar verme yöntemleri birbirleriyle ilişkili çok sayıda kriter ve alternatifin olduğu bir ortamda karar verme sürecini analiz eder. Temel amaç en iyi alternatifi seçmek veya verilen çok sayıda alternatifin performans sıralanmasını belirlemektir. Karar verme bir karar vericinin belirli bir konuda genellikle çelişen kriterler arasından seçim yapmasıdır. Başka bir ifadeyle bir problemin çözümünde harekete geçmektir.

Bu yöntemler karar vericiye en iyi çözümü bulma konusunda yol gösterir. Bunu yaparken ideal çözümler ile ideal olmayan çözümleri karşılaştırırlar. Çok sayıda yöntem olmasına rağmen her zaman bir optimal çözüme ulaşmak mümkün değildir. Bu çerçevede, yöntemlerin güvenirliği konusunda duyarlılık analizleri önerilmektedir. Ayrıca, yapılan çalışmalar göstermektedir ki çok kriterli karar verme yöntemlerin sonuçları arasında yüksek düzeyde istatistiksel olarak anlamlı ilişkiler göstermektedir. Bu durum karar vericilerin amaçlarına yönelik olarak hangi yöntemin seçilmesi problemini ortadan kaldırmaktadır.

COPRAS yöntemi geniş bir alanda stratejik kararların çözümünde kullanılmaktadır. Yöntem, nicel ve nitel kriterleri aynı anda kullanabilir. Değerlendirme sürecinde maksimum ve 
minimum kriterleri ayrı şekilde hesaplayabilir. COPRAS yöntemindeki temel amaç karşılaştırmalı endekslerden boyutsuz ağırlık değerleri hesaplayarak tüm nitelikleri karşılaştırılabilir hale getirmesidir. Duyarlılık analizi, yöntemin güvenilir olduğunu göstermektedir. COPRAS yöntemi, karar vericiye tüm alanlarda kullanılabilir bir esneklik sağlamaktadır.

\section{KAYNAKÇA}

Adali, E. A., \& Işı1k, A. T. (2016). Air conditioner selection problem with COPRAS and ARAS methods. Manas Sosyal Araştırmalar Dergisi, 5(2), 124-138.

Aghdaie, M. H., Zolfani, S. H., \& Zavadskas, E. K. (2013). Market segment evaluation and selection based on application of fuzzy AHP and COPRAS-G methods. Journal of Business Economics and Management, 14(1), 213-233. doi: $10.3846 / 16111699.2012 .721392$

Amoozad Mahdiraji, H., Arzaghi, S., Stauskis, G., \& Zavadskas, E. K. (2018). A hybrid fuzzy BWM-COPRAS method for analyzing key factors of sustainable architecture. Sustainability, 10(5), 1626. doi:10.3390/su10051626

Ayçin, E., (2020). Çok Kriterli Karar Verme Bilgisayar Uygulamalı Çözümler. Nobel Yayıncılık. 2. Basım. Ankara

Bakhouyi, A., Dehbi, R., \& Talea, M. (2016, December). Multiple criteria comparative evaluation on the interoperability of LMS by applying COPRAS method. In 2016 Future Technologies Conference (FTC) (pp. 361-366). IEEE.

Barysienè, J. (2012). A multi-criteria evaluation of container terminal technologies applying the COPRAS-G method. Transport, 27(4), 364-372. doi:10.3846/16484142.2012.750624

Bitarafan, M., Zolfani, S. H., Arefi, S. L., \& Zavadskas, E. K. (2012). Evaluating the construction methods of cold-formed steel structures in reconstructing the areas damaged in natural crises, using the methods AHP and COPRAS-G. Archives of Civil and Mechanical Engineering, 12, 360-367. .doi.org/10.1016/j.acme.2012.06.015

Chatterjee, P., Athawale, V. M., \& Chakraborty, S. (2011). Materials selection using complex proportional assessment and evaluation of mixed data methods. Materials \& Design, 32(2), 851-860. doi:10.1016/j.matdes.2010.07.010

Darko, A. P., \& Liang, D. (2020). An extended COPRAS method for multiattribute group decision making based on dual hesitant fuzzy Maclaurin symmetric mean. International Journal of Intelligent Systems, 35(6), 1021-1068. doi. 10.1002/int.22234

Das, M. C., Sarkar, B., \& Ray, S. (2012). A framework to measure relative performance of Indian technical institutions using integrated fuzzy AHP and COPRAS methodology. Socio-Economic Planning Sciences, 46(3), 230-241. doi:10.1016/j.seps.2011.12.001

Dhiman, H. S., \& Deb, D. (2020). Fuzzy TOPSIS and fuzzy COPRAS based multi-criteria decision making for hybrid wind farms. Energy, 202, 117755. doi.org/10.1016/j.energy.2020.117755

Fouladgar, M. M., Yazdani-Chamzini, A., Lashgari, A., Zavadskas, E. K., \& Turskis, Z. (2012). Maintenance strategy selection using AHP and COPRAS under fuzzy 
environment. International Journal of Strategic Property Management, 16(1), 85-104. doi:10.3846/1648715X.2012.666657

Garg, R., Kumar, R., \& Garg, S. (2018). MADM-based parametric selection and ranking of Elearning websites using fuzzy COPRAS. IEEE Transactions on Education, 62(1), 1118.

Ghorabaee, M. K., Amiri, M., Sadaghiani, J. S., \& Goodarzi, G. H. (2014). Multiple criteria group decision-making for supplier selection based on COPRAS method with interval type-2 fuzzy sets. The International Journal of Advanced Manufacturing Technology, 75(5-8), 1115-1130. doi 10.1007/s00170-014-6142-7

Hashemkhani Zolfani, S., \& Bahrami, M. (2014). Investment prioritizing in high tech industries based on SWARA-COPRAS approach. Technological and Economic Development of Economy, 20(3), 534-553. doi:10.3846/20294913.2014.881435

Kablan, Ü. A., \& Erdoğan, Ü. S. (2021). Mülkiyetine Göre Bankaların Finansal Performaslarının Copras Yöntemi İle Analizi: 1980-2018 Yılları Arası Türk Bankacılık Sektörü Üzerine Bir Araştırma. Mali Çözüm Dergisi/Financial Analysis, 30(163).

Kaklauskas, A., Zavadskas, E. K., Raslanas, S., Ginevicius, R., Komka, A., \& Malinauskas, P. (2006). Selection of low-e windows in retrofit of public buildings by applying multiple criteria method COPRAS: A Lithuanian case. Energy and Buildings, 38(5), 454-462. doi:10.1016/j.enbuild.2005.08.005

Kildienè, S., Kaklauskas, A., \& Zavadskas, E. K. (2011). COPRAS based comparative analysis of the European country management capabilities within the construction sector in the time of crisis. Journal of Business Economics and Management, 12(2), 417-434. doi:10.3846/16111699.2011.575190

Kouchaksaraei, R. H., Zolfani, S. H., \& Golabchi, M. (2015). Glasshouse locating based on SWARA-COPRAS approach. International Journal of Strategic Property Management, 19(2), 111-122. doi:10.3846/1648715X.2015.1004565

Kumari, R., \& Mishra, A. R. (2020). Multi-criteria COPRAS method based on parametric measures for intuitionistic fuzzy sets: application of green supplier selection. Iranian Journal of Science and Technology, Transactions of Electrical Engineering, 44(4), 1645-1662. doi.org/10.1007/s40998-020-00312-w

Kundakc1, N., \& Iş1k, A. (2016). Integration of MACBETH and COPRAS methods to select air compressor for a textile company. Decision Science Letters, 5(3), 381-394. doi: $10.5267 / \mathrm{j} . \mathrm{ds} 1.2016 .2 .003$

Kustiyahningsih, Y., \& Aini, I. Q. (2020, October). Integration of FAHP and COPRAS Method for New Student Admission Decision Making. In 2020 Third International Conference on Vocational Education and Electrical Engineering (ICVEE) (pp. 1-6). IEEE.

Madić, M., Marković, D., Petrović, G., \& Radovanović, M. (2014). Application of COPRAS method for supplier selection. In The Fifth International Conference Transport and Logistics-TIL 2014, Proceedings (pp. 47-50).

Makhesana, M. A. (2015). Application of improved complex proportional assessment (COPRAS) method for rapid prototyping system selection. Rapid Prototyping Journal. doi 10.1108/RPJ-03-2014-0027 
Mercangoz, B. A., Yildirim, B. F., \& Yildirim, S. K. (2020). Time period based COPRAS-G method: application on the Logistics Performance Index. LogForum, 16(2). doi.org/10.17270/J.LOG.2020.432

Mishra, A. R., Rani, P., \& Pardasani, K. R. (2019). Multiple-criteria decision-making for service quality selection based on Shapley COPRAS method under hesitant fuzzy sets. Granular Computing, 4(3), 435-449. doi.org/10.1007/s41066-018-0103-8

Mishra, A. R., Rani, P., Pandey, K., Mardani, A., Streimikis, J., Streimikiene, D., \& Alrasheedi, M. (2020). Novel multi-criteria intuitionistic fuzzy SWARA-COPRAS approach for sustainability evaluation of the bioenergy production process. Sustainability, 12(10), 4155. doi:10.3390/su12104155

Nakhaei, J., Lale Arefi, S., Bitarafan, M., \& Kildienè, S. (2016). Evaluation of light supply in the public underground safe spaces by using of COPRAS-SWARA methods. International Journal of Strategic Property Management, 20(2), 198-206. doi:10.3846/1648715X.2015.1132790

Narayanamoorthy, S., Ramya, L., Kalaiselvan, S., Kureethara, J. V., \& Kang, D. (2020). Use of DEMATEL and COPRAS method to select best alternative fuel for control of impact of greenhouse gas emissions. Socio-Economic Planning Sciences, 100996. doi.org/10.1016/j.seps.2020.100996

Nweze, S., \& Achebo, J. (2021). Comparative Enhancement of Mild Steel Weld Mechanical Properties for Better Performance Using COPRAS-ARAS Method. European Journal of Engineering and Technology Research, 6(2), 70-74. doi.org/10.24018/ejers.2021.6.2.2226

Organ, A., \& Yalçın, E. (2016). Performance evaluation of research assistants by COPRAS method. European Scientific Journal, 12(10), 102-109.

Podvezko, V. (2011). The comparative analysis of MCDA methods SAW and COPRAS. Engineering Economics, 22(2), 134-146. doi.org/10.5755/j01.ee.22.2.310

Popovic, G., Stanujkic, D., \& Stojanovic, S. (2012). Investment project selection by applying copras method and imprecise data. Serbian Journal of Management, 7(2), 257-269. Doi. $10.5937 / \mathrm{sjm} 7-2268$

Rathi, K., \& Balamohan, S. (2017). A mathematical model for subjective evaluation of alternatives in fuzzy multi-criteria group decision making using COPRAS method. International Journal of Fuzzy Systems, 19(5), 1290-1299. doi.10.1007/s40815-0160256-z

Roozbahani, A., Ghased, H., \& Shahedany, M. H. (2020). Inter-basin water transfer planning with grey COPRAS and fuzzy COPRAS techniques: A case study in Iranian Central

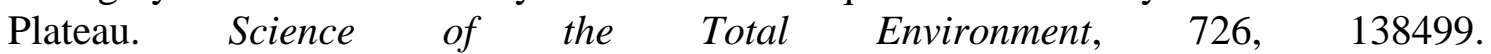
doi.org/10.1016/j.scitotenv.2020.138499

Sałabun, W., Wątróbski, J., \& Shekhovtsov, A. (2020). Are MCDA Methods Benchmarkable? A Comparative Study of TOPSIS, VIKOR, COPRAS, and PROMETHEE II Methods. Symmetry, 12(9), 1549. doi:10.3390/sym12091549

Sarıçalı, G., \& Kundakcı, N. (2016). AHP ve COPRAS yöntemleri ile otel alternatiflerinin değerlendirilmesi. International Review Of Economics And Management, 4(1), 45-66. doi 10.18825/irem.00736 
Turanoglu Bekar, E., Cakmakci, M., \& Kahraman, C. (2016). Fuzzy COPRAS method for performance measurement in total productive maintenance: a comparative analysis. Journal of Business Economics and Management, 17(5), 663-684. doi:10.3846/16111699.2016.1202314

Valipour, A., Yahaya, N., Md Noor, N., Antuchevičienė, J., \& Tamošaitienė, J. (2017). Hybrid SWARA-COPRAS method for risk assessment in deep foundation excavation project: An Iranian case study. Journal of Civil Engineering and Management, 23(4), 524-532. doi.org/10.3846/13923730.2017.1281842

Vytautas, B., Marija, B., \& Vytautas, P. (2015). Assessment of neglected areas in Vilnius city using MCDM and COPRAS methods. Procedia Engineering, 122, 29-38. doi: 10.1016/j.proeng.2015.10.004

Wang, Z. L., You, J. X., Liu, H. C., \& Wu, S. M. (2017). Failure mode and effect analysis using soft set theory and COPRAS method. International Journal of Computational Intelligence Systems, 10(1), 1002-1015.

Yazdani, M., Alidoosti, A., \& Zavadskas, E. K. (2011). Risk analysis of critical infrastructures using fuzzy COPRAS. Economic research-Ekonomska istraživanja, 24(4), 27-40.

Yazdani, M., Jahan, A., \& Zavadskas, E. (2017). Analysis in Material Selection: Influence of Normalization Tools on COPRAS-G. Economic Computation \& Economic Cybernetics Studies \& Research, 51(1).

Zagorskas, J., Burinskienè, M., Zavadskas, E., \& Turskis, Z. (2007). Urbanistic assessment of city compactness on the basis of GIS applying the COPRAS method. Ekologija, 53.

Zavadskas, E. K., Kaklauskas, A., Peldschus, F., \& Turskis, Z. (2007). Multi-attribute assessment of road design solutions by using the COPRAS method. The Baltic Journal of Road and Bridge Engineering, 2(4), 195-203.

Zavadskas, E. K., Turskis, Z., Tamosaitiene, J., \& Marina, V. (2008). Selection of construction project managers by applying COPRAS-G method. Computer Modelling and New Technologies, 12(3), 22-28.

Zavadskas, E.K., Kaklauskas, A., \& Sarka, V., (1994) . The new method of multicriteria complex proportional assessment of projects. Technol. Econ. Dev. Econ. 1 (3), 131-139.

Zheng, Y., Xu, Z., He, Y., \& Liao, H. (2018). Severity assessment of chronic obstructive pulmonary disease based on hesitant fuzzy linguistic COPRAS method. Applied Soft Computing, 69, 60-71. doi.org/10.1016/j.asoc.2018.04.035

Zolfani, S. H., Rezaeiniya, N., Aghdaie, M. H., \& Zavadskas, E. K. (2012). Quality control manager selection based on AHP-COPRAS-G methods: a case in Iran. Economic $\begin{array}{lll}\text { research-Ekonomska istraživanja, } & \text { 25(1), }\end{array}$ doi.org/10.1080/1331677X.2012.11517495 
Ek 1- Performans Sonuçları

Tablo 10: Batı Avrupa: Şehre Göre Mevcut Yaşam Kalitesi İndeksi

\begin{tabular}{|c|c|c|}
\hline Sira & Kent & Yaşam Kalitesi İndeksi \\
\hline 1 & Zürih, İsviçre & 194,86 \\
\hline 2 & Lahey (Den Haag), Hollanda & 191,85 \\
\hline 3 & Eindhoven, Hollanda & 186,08 \\
\hline 4 & Cenevre, İsviçre & 185,88 \\
\hline 5 & Viyana, Avusturya & 183,89 \\
\hline 6 & Lüksemburg, Lüksemburg & 181,96 \\
\hline 7 & Münih, Almanya & 176,18 \\
\hline 8 & Düsseldorf, Almanya & 175,40 \\
\hline 9 & Frankfurt, Almanya & 172,30 \\
\hline 10 & Rotterdam, Hollanda & 169,52 \\
\hline 11 & Amsterdam, Hollanda & 167,94 \\
\hline 12 & Stuttgart, Almanya & 165,62 \\
\hline 13 & Hamburg, Almanya & 163,13 \\
\hline 14 & Berlin, Almanya & 158,48 \\
\hline 15 & Cologne, Almanya & 157,56 \\
\hline 16 & Antwerp, Belçika & 151,00 \\
\hline 17 & Lyon, Fransa & 148,83 \\
\hline 18 & Brüksel, Belçika & 140,98 \\
\hline 19 & Paris, Fransa & 117,88 \\
\hline
\end{tabular}

Kaynak: Numbeo, 2021 
Ekonomi, İsletme ve Maliye Araştırmaları Dergisi, Cilt 3, Sayı 2, s. 136-155

Ek 1 Devamı- Performans Sonuçları

Tablo 11: COPRAS Yöntemi Yaşam Kalitesi Sıralaması

\begin{tabular}{|c|c|c|}
\hline Sura & Kent & $\mathbf{P i}$ \\
\hline 1 & Lahey (Den Haag), Hollanda & 100,00 \\
\hline 2 & Eindhoven, Hollanda & 96,70 \\
\hline 3 & Viyana, Avusturya & 90,14 \\
\hline 4 & Zürih, İsviçre & 89,34 \\
\hline 5 & Düsseldorf, Almanya & 88,21 \\
\hline 6 & Cenevre, İsviçre & 87,32 \\
\hline 7 & Rotterdam, Hollanda & 87,11 \\
\hline 8 & Frankfurt, Almanya & 86,69 \\
\hline 9 & Lüksemburg, Lüksemburg & 86,59 \\
\hline 10 & Amsterdam, Hollanda & 83,73 \\
\hline 11 & Stuttgart, Almanya & 82,45 \\
\hline 12 & Münih, Almanya & 82,16 \\
\hline 13 & Hamburg, Almanya & 81,43 \\
\hline 14 & Berlin, Almanya & 80,37 \\
\hline 15 & Cologne, Almanya & 79,75 \\
\hline 16 & Antwerp, Belçika & 79,05 \\
\hline 17 & Lyon, Fransa & 76,98 \\
\hline 18 & Brüksel, Belçika & 75,40 \\
\hline 19 & Paris, Fransa & 63,84 \\
\hline
\end{tabular}


Ek 2- Extended Summary

\title{
Application of the Copras Method
}

\author{
in the Decision-Making Process
}

Individuals, investors and companies make decisions throughout their lives. A value is created when the benefit of these decisions exceeds the cost incurred. Every decision made represents a solution to a problem encountered. If the real world were operating under the assumption of complete information, every decision made would be correct and daily life would consist of optimal choices. On the other hand, daily life operates under the reality of incomplete information. Each new information coming to the market explains the pricing of the assets. This information is sometimes perceived positively by the market, and sometimes negatively. Market players take positions depending on these risk factors.

Individuals and companies have to analyze many alternatives and criteria in the decisionmaking process. Multi-Criteria Decision Making (MCDM) methods are applied in a decision problem consisting of many alternatives and criteria. These methods do not always offer an optimal solution. However, they minimize possible decision errors.

COmplex Proportional ASsessment (COPRAS) is one of the multi-criteria decision making methods. According to (Podvezko, 2011; Das, 2012; Amoozad Mahdiraji, et al., 2018; Roozbahani, et al., 2020), it selects the best decision alternatives by considering ideal and worst-ideal solutions. It applies a gradual ranking and evaluation procedure in terms of importance and degree of benefit of alternatives. A multi-criteria evaluation approach is used to both maximize and minimize criterion values. The method examines the effect of maximizing and minimizing criteria on the evaluation result. The selection of the best alternative is made by considering both ideal and non-ideal solutions. Selects the best scenario among the available scenarios.

The reliability and accuracy of his method are accepted by many scientists and are now used to solve multi-specific problems in different engineering and management fields. The calculation steps and time of the method are short. The COPRAS method was introduced to the literature by (Zavadskas, et al., 1994).

The aim of the study is to apply the COPRAS method in the decision making process. A case study has been conducted for this. Western Europe: Current Quality of Life by City Index was used to demonstrate the application. Quality of Life Index consists of eight criteria. These criteria are Purchasing Power Index, Safety Index, Health Index, Climate Index, Cost of Living Index, Property Price Income Ratio, Traffic Commencement Time Index and Pollution Index. These criteria used in the Quality of Life Index calculation are classified as higher is better and lower is better.

During the implementation process, the direction of the criteria was determined as maximum for Purchasing Power Index (C1), Safety Index (C2), Health Index (C3) and Climate Index (C4). Other criteria; Cost of Living Index (C5), Property Price Income Ratio (C6), Traffic Commencement Time Index (C7) and Pollution Index (C8) are set as the minimum. The aim is to choose cities and cities with the highest Quality of Life Index.

For the mathematical representation of the COPRAS Method (Wang, et al., 2017, p. 1007), his study was used. The study (Ayçin, 2020, p. 67-71) was used to solve the COPRAS method. The method takes place in five stages after the decision matrix is created.

According to the results of the COPRAS method for evaluating the current quality of life by Western European city, the top five cities with the best quality of life are respectively The 
Hague (Den Haag), Eindhoven, Vienna, Zurich, and Düsseldorf. The last five cities are Cologne, Antwerp, Lyon, Brussels and Paris respectively. The results are consistent according to the Quality of Life Index order.

In this part of the study, sensitivity analysis of the results was made. For this, the correlation relationship of nonparametric series was examined using Kendall's tau_b and Spearman's rho approximations. Table 10 shows the relationship between Quality of Life Index and COPRAS method performance results.

According to Table 10, there is a strong positive relationship between Quality of Life Index and COPRAS method performance results. Correlations are statistically significant. This result provides an important evidence about the reliability of studies conducted with the COPRAS method.

Multi-criteria decision-making methods analyze the decision-making process in an environment where there are many interrelated criteria and alternatives. The main purpose is to select the best alternative or to determine the performance ranking of a large number of alternatives given. Decision making is when a decision maker chooses among often conflicting criteria on a particular issue. In other words, it is taking action to solve a problem.

These methods guide the decision maker to find the best solution. In doing so, they compare ideal solutions with non-ideal solutions. Although there are many methods, it is not always possible to reach an optimal solution. In this context, sensitivity analyzes are recommended for the reliability of the methods. In addition, studies show that there is a high level of statistically significant relationships between the results of multi-criteria decision making methods. This situation eliminates the problem of choosing which method for the purposes of decision makers.

The COPRAS method is used in a wide range of strategic decisions. The method can use quantitative and qualitative criteria simultaneously. Can calculate the maximum and minimum criteria separately in the evaluation process. The main purpose of the COPRAS method is to make all attributes comparable by calculating dimensionless weight values from comparative indices. Sensitivity analysis shows that the method is reliable. The COPRAS method provides the decision maker with flexibility that can be used in all areas. 